Mots. Les langages du politique

\title{
Les héméronymes. Ces évènements qui font date, ces dates qui deviennent évènements
}

\author{
Laura Calabrese Steimberg
}

\section{CpenEdition}

Journals

Édition électronique

URL : https://journals.openedition.org/mots/14443

DOI : $10.4000 /$ mots. 14443

ISSN : 1960-6001

Éditeur

ENS Éditions

Édition imprimée

Date de publication : 1 novembre 2008

Pagination : 115-128

ISBN : 978-2-84788-147-9

ISSN : 0243-6450

Référence électronique

Laura Calabrese Steimberg, « Les héméronymes. Ces évènements qui font date, ces dates qui deviennent évènements », Mots. Les langages du politique [En ligne], 88 | 2008, mis en ligne le 01 novembre 2010, consulté le 22 avril 2022. URL : http://journals.openedition.org/mots/14443; DOI : https://doi.org/10.4000/mots.14443

Ce document a été généré automatiquement le 22 avril 2022.

(c) ENS Éditions 


\title{
Les héméronymes. Ces évènements qui font date, ces dates qui deviennent évènements
}

\author{
Laura Calabrese Steimberg
}

1 Les médias nous confrontent tous les jours à des désignations d'évènements ; une fois installées, celles-ci constituent le moyen le plus économique pour référer aux faits, discours, croyances et lieux communs qui constituent pour nous l'évènement, et assurer sa reconnaissance tout au long de sa permanence dans l'espace médiatique. Le 11 septembre, l'Intifada, l'affaire du voile ou bien Outreau sont des prêts-à-dire, des éléments du discours servant à désigner des pans entiers de notre contexte situationnel et, ce faisant, à le construire en même temps. Ils sont le produit d'un protocole de nomination journalistique qui a des conséquences dans notre perception de l'évènement, et qu'il est nécessaire de considérer comme une pratique globale de la presse écrite ${ }^{1}$. Dans la mesure où ils établissent avec leur objet un type de référence désignative, nous avons choisi de les appeler désignants évènementiels.

Ces désignants évènementiels sont polyréférentiels : ils réfèrent à une date, à un objet concret ou abstrait ou bien à un lieu, mais dans leur emploi médiatique, leur référent est un ensemble de faits d'actualité que nous identifions comme un évènement ${ }^{2}$. Une fois lancé dans l'espace médiatique, le désignant va isoler une ou (tout au plus) deux coordonnées de l'évènement, effaçant les autres au profit de l'économie linguistique. Par exemple, les toponymes évènementiels (Tchernobyl) souligneront l'endroit où l'évènement $\mathrm{a}$ eu lieu, les xénismes (tsunami) le ramèneront indirectement à ses coordonnées toponymiques à travers la consonance phonétique, et les mots-évènements stricts ${ }^{3}$ récupéreront la nature des faits grâce à leur contenu sémantique («affaire ", "guerre ", " catastrophe »...). Quant aux autres coordonnées de l'évènement (quoi, comment, etc.), elles sont contenues implicitement dans le désignant.

Parmi les désignants évènementiels, il y en a un qui met en avant les coordonnées temporelles et que nous proposons d'appeler héméronyme (du grec héméra: jour) ${ }^{4}$; il s'agit d'une catégorie peu étudiée, dont le paradigme est pour nous le 11 septembre, qui 
a une présence importante dans le discours médiatique et dans notre perception de la réalité politique actuelle. Face à une terminologie hésitante, nous avons pour but de décrire ce type de désignants en proposant un nouveau concept.

Dans un article de 1996, Büchi (1996, p. 271) propose le terme de chrononyme pour désigner les «noms propres de temps », concept développé par Van de Velde (2000) qui $\mathrm{y}$ voit une projection naturelle de la triade déictique sur laquelle repose la référence (à côté des noms propres de personne et de lieu). Elle classe dans cette catégorie les noms des jours, des années, des mois et les structures syntaxiques équivalentes, et remarque que «les noms propres de temps, comme ceux des lieux, peuvent devenir aussi un moyen de référer à des évènements particuliers: le 4juillet, octobre 17, Septembre noir» (Van de Velde, 2000, p.44). Pour sa part, Flaux considère comme des noms propres de temps Mai 68 et Saint-Barthélémy (Flaux, 2000, p. 123), tandis que Leroy (2004, p. 173) hésite pour ce dernier entre chrononyme et praxonyme, c'est-à-dire entre nom propre de temps et nom de fait historique. La catégorie de praxonyme est d'ailleurs loin de faire l'unanimité : pour Bauer (1985), qui a proposé le concept, il comprend des faits historiques, des évènements culturels et des noms de maladies, mais d'autres chercheurs (Daille, Fourour, Morin, 2000, p. 122) proposent d'y ajouter les noms de périodes historiques (le Paléolithique).

5 Le statut avéré de nom propre mis à part, ces hésitations posent le problème de la durée du fait désigné (évènement ponctuel ou période ?) et des caractéristiques morphosémantiques du nom (quels sens véhicule-t-il selon qu'il s'agit d'un nom propre ou d'une date ?). Pour éviter le flou terminologique, nous réserverons le concept de chrononyme aux périodes historiques, et celui d'héméronyme aux dates qui constituent des noms d'évènements 5 .

6 Nous proposons d'aborder les héméronymes dans le cadre d'une recherche plus large portant sur la dénomination médiatique, en nous focalisant non pas sur l'évènement luimême mais sur la matérialité de l'expression servant à le nommer ; nous nous occuperons ainsi des mots et non pas des référents (en classant les évènements selon leur nature linguistique et non pas ontologique $\left.{ }^{6}\right)$, pour aborder les héméronymes dans leur spécificité morphosyntaxique, sémantique et pragmatique afin de saisir leur fonctionnement discursif ${ }^{7}$.

7 Pour exposer notre analyse de cette catégorie, nous allons aborder le système global des désignants évènementiels, dans lequel les héméronymes sont un élément d'un ensemble plus large ${ }^{8}$. En raison de leur proximité avec le nom propre, ils sont à mettre en relation avec les toponymes évènementiels, et en tant que désignants de temporalité, ils doivent être mis en série avec les chrononymes. Nous essaierons de décrire leur spécificité à partir d'une observation du corpus proposé, pour ensuite aborder la question de leur motivation, autrement dit, pour voir s'il y a corrélation entre le type d'évènement (politique, climatique, etc.) et le type de désignant (toponyme évènementiel, xénisme, etc.).

8 En ce qui concerne les exemples analysés, ils sont tirés d'un corpus ouvert, qui croît au fil des jours et classé selon deux critères : par type de désignant et par évènement. Le premier classement nous permet d'observer le comportement morphosyntaxique et sémantique de chaque catégorie par rapport aux autres; le deuxième nous permet de saisir l'émergence d'un désignant évènementiel ainsi que son évolution, c'est-à-dire ses formes de circulation et le processus de figement, sa disparition et réapparition dans l'espace public'. 


\section{Caractéristiques morphosyntaxiques et sémantiques}

9 L'héméronyme est une date qui désigne un évènement. Il faut distinguer les deux usages, car la date et le nom de l'évènement ont un comportement syntaxique spécifique. Le même syntagme peut être utilisé alternativement comme date (1) tout en se référant à l'évènement en question, ou comme héméronyme (2) :

(1) La publicité se fera discrète aux États-Unis le 11 septembre. (lemonde.fr, 8 septembre 2002)

United Airlines et American Airlines vont suspendre toute campagne ce jour-là.

(11 septembre 2002)

(2) La vérité sur le 11 mars - Le 11 mars dernier, l'Espagne a subi le pire attentat de son histoire, l'un des plus sanglants que le monde ait connus. (lemonde.fr, 31 mars 2004)

10 L'héméronyme peut tout aussi bien être le nom d'une année, mais dans ce cas, sa circulation est forcément plus restreinte car il a besoin d'un contexte fort pour être actualisé en tant que désignant évènementiel, comme dans l'exemple (3) tiré d'un dossier sur la Guerre des six jours :

(3) Les deux évènements qui continuent à structurer les consciences dans le monde arabe sont sûrement 1948 et 1967. (Courrier international, 31 mai-6 juin 2007)

11 Il peut aussi y avoir des usages doubles, où date et héméronyme se confondent (dans ce cas, grâce à la typographie) :

(4) M. Sarkozy ne donnera finalement pas de conférence de presse le 14-Juillet.

(lemonde.fr, 5 juillet 2007)

12 En tant que date (usages attestés mais rares), le syntagme remplit la fonction de circonstanciel de temps et il sert le plus souvent soit à situer l'évènement ( 5 ), soit à le narrativiser (6) :

(5) Lloyd's de Londres: appel pour le financement des indemnités liées à la destruction du WTC, World Trade Center, le 11 septembre 2001. (lemonde.fr, 8 février 2002)

(6) New York, mardi 11 septembre, 9 h 03. (lemonde.fr, 16 septembre 2001)

En fonction évènementielle (héméronyme), la date peut être accompagnée d'un article défini, mais celui-ci est souvent effacé lorsqu'il apparait en position thématique :

(7) 11 septembre 2001: Mme Rice défend pied à pied l'administration Bush.

(lemonde.fr, 9 avril 2004)

Cette élision est due à un processus d'effacement qui touche tous les désignants évènementiels, en raison de leur énorme pouvoir évocateur et de leur capacité à condenser toutes les coordonnées évènementielles sans devoir les rappeler à chaque occurrence du terme. Même s'il s'agit d'un phénomène très stéréotypé dans la presse, qui répond à un besoin d'économie linguistique plutôt qu'à une visée stylistique, nous y voyons un type de métonymie qui produirait un effet très particulier sur la représentation de l'évènement. Sur le modèle de la métonymie, nous postulons un déplacement de la date à laquelle l'évènement a eu lieu vers l'évènement lui-même. Comme pour toute métonymie, «il s'agit d'une modification qui intervient sur l'axe syntagmatique tout en provoquant un transfert de référence » (Le Guern, 1973, p. 28), qui investirait la date d'une certaine autonomie sémantique et syntaxique.

Bien entendu, l'héméronyme ne nait pas tel quel, prêt à circuler ; il est l'aboutissement d'un processus de condensation du sens et du syntagme où intervient l'économie 
propre au discours journalistique, et qui se traduit sous la forme de titres bisegmentaux à deux points (Bosredon, Tamba, 1992), comme dans l'exemple (7).

Lorsqu'on retrace ce processus de condensation, il est toujours possible de retrouver les éléments qui ont été effacés, et notamment le nom qui décrit la nature de l'évènement, de sorte que le désignant résultant n'est finalement, du point de vue syntaxique, qu'un complément déterminatif de ce nom. Dans 11 mars et 11 septembre, le nom effacé est « attentat(s) », comme « catastrophe » pour Tchernobyl, « affaire » pour le voile, etc.

Nous pourrions émettre l'hypothèse que c'est ce nom-évènement strict qui nous permet d'actualiser ces syntagmes hypercondensés comme des désignants évènementiels. L'avantage de cette catégorie (les noms-évènements stricts, qui doivent toujours s'associer avec des compléments pour constituer un désignant évènementiel) est de révéler le mécanisme de condensation qui affecte tous les désignants évènementiels, en repérant les éléments effacés au fur et à mesure que se construit le désignant d'évènement, et qui ressortent de temps en temps dans les titres de presse, comme dans (8) :

(8) Attentat du 11 mars : le ministère de l'Intérieur a ignoré les menaces sur le chef

du commando. (lemonde.fr, 18 octobre 2004)

18 Nous ne parlons ici que des éléments linguistiques marqués, car le désignant évènementiel est aussi composé d'une série d'éléments épilinguistiques (lieux communs, images, idées reçues, etc.), que nous appelons le sens satellitaire et qui se construit autour d'une désignation constituante.

$\mathrm{Du}$ point de vue sémantique, il est intéressant de noter que, dans le cas des héméronymes (comme dans celui des toponymes évènementiels), c'est la composante la plus informative qui disparait (« attentat»), mais paradoxalement, c'est cela qui permet de spécialiser la référence (il y a beaucoup d'attentats dans notre actualité, mais statistiquement, il est peu probable qu'ils arrivent à la même date, si bien que la date finit par être plus informative que le nom). Dans le cas du 21 avril, c'est bien entendu le terme « élections » qui a été effacé, mais les sens satellitaires ont tellement pris le dessus par rapport à la désignation constituante qu'ils ont occulté la nature initiale de l'évènement ; cependant, celle-ci ressort souvent dans le contexte de nombreux titres de presse, l'expression étant utilisée pour faire référence à des élections :

(9) Le 21 avril 2002 pèse sur la campagne - Le référendum du 29 mai prochain sera-

t-il une réplique du séisme du 21 avril 2002 ? (lenouvelobs.com, 25 avril 2005)

\section{L'héméronyme par rapport aux autres désignants évènementiels}

Tous ces sens effacés ne nous empêchent pas d'actualiser le syntagme comme un désignant évènementiel ; cependant, il est légitime de nous demander quelles sont les conséquences, du point de vue de notre représentation de l'évènement, du fait que la désignation ne garde que ses coordonnées temporelles. Autrement dit, considérant que les deux grandes coordonnées de l'évènement sont le temps et l'espace, pourquoi certains se déclinent-ils à partir de l'une ou l'autre ? Y a-t-il un lien motivé entre une catastrophe naturelle et le fait de l'appeler par un xénisme (le tsunami), entre une catastrophe provoquée par l'erreur humaine et un toponyme (Tchernobyl) ? 
21 Prenons une seule catégorie désignative, les toponymes évènementiels, et comparons les évènements auxquels ils réfèrent: Tchernobyl, Auschwitz, Outreau, (le) Heyzel. Ils peuvent désigner des évènements politiques ou des faits de société de teneurs très différentes : une catastrophe nucléaire, une catastrophe sanitaire, la solution finale (et la guerre, le nazisme, l'horreur concentrationnaire..., les limites connotatives n'étant pas établies dans la désignation), un procès pour pédophilie (et les dysfonctionnements de la justice française) ou un fait divers qui devient débat de société comme le hooliganisme. Rien ne nous permet de dire que la nature des référents motive le choix du désignant. Seules certaines catégories d'évènements ont recours systématiquement à un type de désignant, comme par exemple les batailles et les phénomènes atmosphériques (rebaptisés phénonymes dans la classification de Bauer), choix qui garde vraisemblablement un lien avec le genre du discours (historique, météorologique) et les disciplines qui les produisent ${ }^{10}$.

Dans le cas des toponymes évènementiels, donc, la motivation entre la morphologie du désignant et le type d'évènement est tout à fait obscure. Dans le pôle opposé, nous trouvons les noms d'évènements météorologiques (la canicule) et les mots évènements stricts, qui décrivent la nature de l'évènement ${ }^{11}$ et s'actualisent à l'aide d'un complément, généralement de temps ou d'espace (la guerre de Kippour, la crise des banlieues) mais aussi des lexèmes plus descriptifs (la crise de la vache folle, l'affaire du voile). Pour ce qui est des xénismes, ce sont des objets mixtes étant donné que leur transparence dans les langues d'origine est complètement effacée dans la langue d'emprunt : ainsi pour le tsunami et l'Intifada, réinvestis pour nous des caractéristiques des évènements qu'ils désignent mais sans valeur en langue.

La question de la motivation du désignant est en rapport avec sa capacité descriptive, et en conséquence avec sa charge sémantique, comme le montre le schéma suivant :

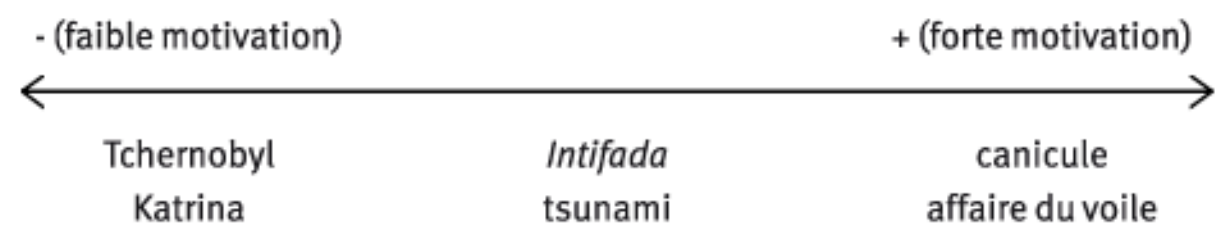

24 Ce schéma souligne notamment le rapport du désignant avec un référent unique, et en conséquence avec le nom propre. Ainsi, les mots-évènements stricts désignent, le temps que dure le moment discursif (Moirand, 2007), un évènement unique : il n'y a qu'une seule canicule, une seule affaire du voile, un seul tsunami et une seule Intifada au moment où la presse désigne l'évènement ${ }^{12}$. C'est le pouvoir déictique des désignants évènementiels, leur capacité à faire référence au contexte situationnel qui nous permet d'actualiser correctement la désignation et de ne pas mélanger les moments discursifs (la première et la deuxième Intifada, l'affaire du voile de 1989 et celle de 2004, la canicule de 2003 et celles qui l'ont précédée).

Mais c'est le comportement des xénismes qui est particulièrement éclairant, car ils exemplifient ce voyage entre nom propre et nom commun qui caractérise ces objets discursifs. En effet, si, dans la langue d'origine, ils constituent un nom commun, dans la langue d'emprunt, ils vont se spécialiser pour désigner non pas un évènement 
générique, mais un évènement aux coordonnées bien définies. Par la suite, ils peuvent « revenir » à la langue d'emprunt après un premier passage par des usages purement discursifs, sous la forme d'une entrée du dictionnaire, comme c'est le cas pour tsunami, devenu nom commun en français ${ }^{13}$.

Ce qui nous amène aux héméronymes : où les placer dans notre schéma? Quel est leur rapport au nom propre? Leur morphologie motive-t-elle leur usage par rapport à un certain type d'évènements?

\section{Caractéristiques pragmatiques}

27 Au-delà de sa forme particulière, l'héméronyme a un comportement semblable au toponyme évènementiel; le fait qu'il ait une faible valeur en langue renforce l'impression d'unicité de sa relation référentielle et le rapproche du nom propre, ce qui pose quelques particularités au niveau de la référence. Contrairement à d'autres désignants évènementiels, comme les xénismes ou les désignants météorologiques, qui s'actualisent uniquement en fonction du contexte (le tsunami ou la canicule sont toujours les derniers en date), les héméronymes et les toponymes évènementiels ont la capacité de bloquer toute autre référence mémorielle (même si, comme on le verra plus loin, les statistiques peuvent parfois enfreindre cette tendance). En effet, l'observation du corpus vient confirmer l'intuition théorique : les dates en fonction évènementielle désignent toujours des évènements qui, précisément, "font date ", qui marquent un avant et un après.

Dans ce sens, l'effet «rupture de série » rend l'héméronyme apte à désigner des évènements plus ou moins limités dans le temps, à forte composante politique et qui interrompent le déroulement habituel d'une société. À tel point qu'ils peuvent même désigner un évènement qui n'a pas eu lieu, mais dont la nature brutale et inespérée exige un acte de nomination, comme dans l'exemple (10), tiré d'un quotidien britannique du 11 août 2006, un jour après que la police a déjoué des attentats terroristes :

(10) 10/8 Was this going to be the next date in the calendar of terror? [Le 10/8 allait-il

être la prochaine date sur le calendrier de la terreur ?] (The Independent)

Les héméronymes auraient ainsi un effet très particulier sur la construction de l'évènement, interrompant une temporalité naturelle et instaurant une série transversale de faits qui, cependant, ne sont pas perçus de manière homogène (ainsi pour le 11 septembre ou le 21 avril, qui dépassent le fait unique pour désigner une conjoncture politique).

Si tous les désignants évènementiels, en tant que désignations hypersynthétiques, tendent à effacer la plupart des coordonnées évènementielles en faveur de l'économie linguistique, les héméronymes vont notamment effacer la durée de l'évènement; leur fonctionnement est dans ce sens à l'opposé des chrononymes comme désignations de périodes, même si, du point de vue tant ontologique que phénoménologique, l'évènement en question a aussi une profondeur temporelle et peut même être fortement narrativisé, comme ce fut le cas pour le 11 septembre (et notamment sa mise en récit par la télévision).

31 L'héméronyme désigne aussi, comme tous les désignants évènementiels, une famille de faits qui s'étendent dans le temps, et non un évènement unique et bien délimité, 
puisque par définition l'évènement n'est jamais homogène et ponctuel; d'une certaine façon, il est toujours période. Cependant, sa morphologie et son faible contenu sémantique, qui le prédispose aux relations désignatives plus que nominatives, effacent la durée de l'évènement, contrairement à d'autres désignants évènementiels qui portent en eux des sèmes de temporalité ("conflit», "guerre»), ou à ceux qui l'ignorent par simple omission, comme les toponymes évènementiels. Autrement dit, il balise lui-même sa propre durée (très brève), commence par une irruption (ou du moins par un fait qui est perçu comme tel) et institue sa propre date de commémoration.

Cet effet de rupture se trouve déjà, et avant tout, dans les dates consacrées par le discours des historiens :

Dans cette perspective émancipatrice qui est celle des républicains, le 14 juillet 1789, et lui seul, peut délimiter de façon indiscutable un avant et un après irréductibles; tracer une frontière insurmontable entre un Ancien Régime condamné et une France moderne définitivement affranchie, malgré les multiples tentatives réactionnaires $\mathrm{du} \mathrm{xIX}^{\mathrm{e}}$ siècle, du triple joug féodal, clérical et monarchique ; bref, signifier qu'avec la chute de la sombre Bastille médiévale, «c'est le monde ancien qui finit et un monde nouveau qui commence ». (Amalvi, 1997, p. 387)

En effet, ce sont le plus souvent les toponymes évènementiels (11) et les héméronymes $(12,13)$ qui intègrent des constructions du type «l'après $\mathrm{X}$ », et plus rarement « l'avant $\mathrm{X}$ », lesquels contribuent à construire l'évènement comme rupture de l'ordre social :

(11) Vienne : l'après-Tchernobyl. (humanite.fr, 13 avril 1996)

(12) Spécial Espagne. La révolution de l'après 11-mars -

Comme il y a eu aux États-Unis un avant et un après-11 septembre, il y aura en

Espagne un avant et un après-11 mars (lenouvelobservateur.fr, 9 avril 2004)

(13) Paroles frontistes de l'après 21-avril (lemonde.fr, 21 avril 2007)

La notation de ce type de syntagmes, avec un trait d'union la plupart du temps, met en évidence cette problématique ouverte par les héméronymes, à savoir leur statut de noms propres et leur perception en tant que faits uniques. Nous en avons trouvé un exemple très parlant dans le forum de traduction www.wordreference.com, où des traducteurs en sont venus à se demander comment il convenait d'orthographier le syntagme l'après 11 septembre. On y propose la majuscule pour le mois (sur l'exemple de la Révolution), le trait d'union entre après et la date (comme dans l'après-guerre) ou bien entre le jour et le mois (en se basant sur la règle d'écriture des noms de rues). Quoi qu'il en soit, il ne faudrait pas appliquer les règles habituelles pour la notation des dates, étant donné qu'« il ne s'agit pas d'une date, il s'agit d'une locution qui devient nom propre. La révolution est un nom commun, qui ne prend donc pas de majuscule. Pourtant, quand on écrit la Révolution, on sait tout de suite de laquelle on parle » - ou bien parce que "ce n'est plus une date, mais le nom d'un évènement ». Quelle serait dans ce cas la différence avec «le 14 juillet, le 11 novembre, le 8 mai, [qui] sont aussi des évènements »? Réponse : "Ce seraient plutôt des dates auxquelles on célèbre un évènement. Quand on dit "le 14 juillet", c'est du 14 juillet de l'année en cours dont on parle, et pas du 14 juillet 1789 . Quand on dit "le 11 s/Septembre", c'est du 11 septembre 2001 dont on parle. »

Bien que l'héméronyme efface la dimension temporelle de l'évènement grâce à l'effet de rupture qu'il produit, ce n'est que pour l'ouvrir en amont et en aval, en inaugurant une nouvelle ère et en donnant place à une dénomination de type chrononymique ( "l'après $\mathrm{X} »)$, sorte de périodisation après-coup que l'héméronyme permettrait de faire ressortir. Si les toponymes évènementiels peuvent intégrer également ce type de 
construction, les héméronymes le font de manière privilégiée grâce à leur sens, en suivant le comportement des noms propres, qui se transforment facilement en début de période, comme dans (14) et (15) :

(14) L'après-Sharon a déjà commencé (lemonde.fr, 11 janvier 2006)

(15) Cuba et l'après-Castro (lemonde.fr, 5 août 2006)

Bien entendu, tout évènement suppose une rupture dans le fonctionnement d'une société, mais la morphologie de l'héméronyme ne met en avant que cet aspect-là, instaurant un lien entre le type de désignant et le type d'évènement. Les héméronymes inaugurent des périodes parce qu'ils rompent la temporalité habituelle, mais ils le font d'une manière particulière, différente des autres désignants évènementiels. Si la rupture propre à tout évènement permet des constructions du type " après $\mathrm{X}$ » (après la canicule, après la vache folle, après le tsunami), ces après n'impliquent pas une périodisation comme lorsque l'adverbe est nominalisé.

C'est cette capacité à inaugurer des périodes (facilitée par la dimension commémorative de toute date) qui lie l'héméronyme à un certain type d'évènement particulièrement brusque ou perçu comme tel pour le déroulement de la vie publique, et notamment politique (ce qui n'est absolument pas en rapport avec l'importance de l'évènement en question ou sa permanence dans le discours médiatique), et qui explique son caractère paradigmatique et sa tendance à construire des antonomases.

\section{La concurrence des désignants et les désignants paradigmatiques}

En effet, plus que les autres désignants évènementiels, les héméronymes se prêtent volontiers à l'antonomase ${ }^{14}$, comme l'attestent de nombreux exemples. La presse fait souvent appel à ce procédé métaphorique pour créer des familles d'évènements et éveiller des images et des discours servant à interpréter les faits qui font irruption dans notre quotidien :

(16) Ce référendum est une réplique du 21 avril 2002. (lemonde.fr, «Entretien avec Pascal Perrineau, directeur du Centre d'étude de la vie politique française», 30 mai 2005)

(17) Le 11 septembre de l'Europe. (lemonde.fr, 13 mars 2004)

Le statut paradigmatique de l'évènement désigné par un héméronyme ne doit pas, nous l'avons déjà dit, nous leurrer quant à l'importance de l'évènement pour la société qui le subit, malgré le fait que l'évènement aux dimensions planétaires qui a inauguré notre siècle est le 11 septembre. En tant que nom d'évènement qui fait date et qui peut inaugurer une nouvelle étape, l'héméronyme a la possibilité de revenir tous les ans pour nous le rappeler, contrairement à d'autres désignants qui doivent être convoqués par des commémorations le plus souvent officielles ${ }^{15}$. En conséquence, au-delà de sa présence plus ou moins grande dans le discours médiatique et la mémoire collective, la morphologie de l'héméronyme lui permet d'être l'aune qui mesure de nouveaux évènements pour les réinterpréter, comme le montre (18) :

(18) Le Mai 81 de la droite - Il flotte un air de mai 1981 sur la droite française [après la victoire de l'UMP aux législatives]. Cette dernière utilise volontiers cette comparaison, à l'instar d'un Jean-Pierre Raffarin, ravi de prendre une sorte de revanche sur la gauche au bout d'un quart de siècle. (lemonde.fr, 11 juin 2007) 
caractère paradigmatique, son figement au calendrier (il n'est pas nécessaire de trouver une date commémorative car l'évènement et la date de commémoration coïncident) et sa morphologie qui le place très près du nom propre peuvent nous donner l'illusion qu'il s'agit d'un évènement unique. Cependant, l'unicité de la relation référentielle doit être nuancée, essentiellement pour des motifs statistiques. Si l'actualité proche nous offre un exemple d'héméronyme double (le 11 septembre), comme dans (19), il ne s'agit pas du seul cas d'évènement en ayant déplacé un autre (20) :

(19) L'autre 11 septembre, il y a vingt-neuf ans, au Chili. (lemonde.fr, 12 septembre 2002)

(20) Il y a soixante ans, les massacres de Sétif : l'autre 8 mai 1945. (lemonde.fr, 7 mai 2005)

La concurrence des désignants peut être mise en avant délibérément pour rappeler des évènements négligés par la mémoire collective, comme dans (20), et souligner l'oubli dans lequel ils ont été relégués, ou pour faire ressortir leur importance. C'est le cas, par exemple, de l'éditorial d'Ignacio Ramonet paru dans Le Monde diplomatique le 12 septembre 2001, qui avait comme titre "L'autre 11 septembre", décrivant un scénario apocalyptique dont tout laissait penser qu'il faisait allusion aux attentats de New York et Washington, mais qui s'avérait être une description du coup d'État du 11 septembre 1973 au Chili ${ }^{16}$. Comme le montre Julien Fragnon (2007), la concurrence des deux désignants a eu pour conséquence le déplacement du 11 septembre chilien mais lui a donné, par contrecoup, une notoriété médiatique qu'il n'avait pas.

D'autre part, l'oubli d'un évènement, ou les tentatives de certains discours officiels pour l'effacer, ont pu conduire à assigner une date commémorative à un nom d'évènement n'ayant pas vraiment acquis le statut d'héméronyme, comme ce fut le cas pour le massacre d'Algériens à Paris le 17 octobre 1961 (Gaïti, 1994) :

(21) Une station de métro pour se souvenir du «17 octobre $1961 »$. (lemonde.fr, 19 février 2007)

Au bout de ce parcours, nous croyons avoir démontré la nécessité d'une nouvelle catégorie pour décrire les noms d'évènements formés à partir d'une date. En les rapprochant des toponymes évènementiels, nous avons voulu souligner leur nature de noms propres, plus forte que celle des autres désignants évènementiels, et leur motivation par rapport au référent, qui en fait les désignants privilégiés des évènements de la rupture politique. En les plaçant dans le contexte des autres désignants évènementiels, nous avons voulu insister sur le fait que ceux-ci constituent une grille plus ou moins fermée (mais protéique et souvent très imaginative) proposée par le discours médiatique, et que selon la forme qu'ils prennent, ils conditionnent notre perception de l'actualité en privilégiant l'une ou l'autre des coordonnées évènementielles.

Toutes ces observations ouvrent de nouvelles problématiques: d'une part, le statut de nom propre de tous les désignants évènementiels, qui exigerait une analyse capable de décrire le lien à la fois fort et éphémère avec leur référent ; d'autre part, le caractère intrinsèquement mémoriel de certains désignants par rapport à d'autres; et finalement, la question de la motivation des désignations d'évènements, qui façonnent quotidiennement notre mémoire historico-médiatique. 


\section{BIBLIOGRAPHIE}

Amalvi Christian, 1997, « Le 14-juillet. Du Dies irae à Jour de fête », P. Nora éd., Les lieux de mémoire, Gallimard, p. 383-422.

BAUER Gerhard, 1985, Namenkunde des Deutschen, Berne, Germanistische Lehrbuch-sammlung.

BOSREDON Bernard, TAMBA Irène, 1992, "Thème et titre de presse : les formules bi-segmentales articulées par un "deux points" ", L'Information grammaticale, n 54, p. 36-44.

BücHI Eva, 1996, Les structures du « Französiches Etymologisches Wöterbuch ». Recherches métalexicographiques et métalexicologiques, Tübingen, Niemeyer.

CALABRESE Laura, à paraitre/a, « L'émergence du contexte dans les désignants évènementiels : intuition du chercheur ou matérialité discursive ?", colloque Discours et contexte, Université de Montpellier, mai 2007.

- à paraitre/b, « Nommer un évènement ou les marges du sens dans les désignations médiatiques : l'exemple de la canicule », colloque Représentations du sens linguistique 3, Université libre de Bruxelles, novembre 2005.

DAILle Béatrice, Fourour Nordine, Morin Emmanuel, 2000, « Catégorisation des noms propres : une étude en corpus », Cahiers de grammaire, n² 25, p. 115-129.

Flaux Nelly, 2000, « Nouvelles remarques sur l'antonomase », Lexique, n 15, p. 117-144.

FRAGNON Julien, 2007, «Quand le 11-Septembre s'approprie le onze septembre. Entre dérive métonymique et antonomase », Mots. Les langages du politique, n 85, p. 78-90.

GAїтI Brigitte, 1994, « Une manifestation sans suites : le 17 octobre 1961 à Paris », Sociétés contemporaines, $\mathrm{n}^{\circ} 20$, p. 11-37.

LE GUERN Michel, 1973, Sémantique de la métaphore et de la métonymie, http://www.revue-texto.net/ Parutions/LeGuern/LeGuern.html

LEROY Sarah, 2004, De l'identification à la catégorisation : l'antonomase du nom propre en français, Louvain, Peeters.

MOIRAND Sophie, 2007, Les discours de la presse quotidienne. Observer, analyser, comprendre, Paris, PUF. MoLOTCH Harvey, LESTER Marylin, 1996, « Informer : une conduite délibérée. De l'usage stratégique des évènements ", É. Neveu, L. Quéré éd., Réseaux, nº 75, Le temps de l'évènement, vol. 1, http:// www.enssib.fr/autres-sites/reseaux-cnet

MoRIN Edgar, 1972, « Le retour de l'événement », Communications, n 18, p. 7-20.

SCHUBERT Yan, « Le 9 novembre : symbolique et polysémie d'une date historique », http:// www.lecourrier.ch/index.php?name=NewsPaper\&file=article\&sid=40374

MORTUREUX Marie-Françoise, 1993, « Paradigmes désignationnels », Semen, nº 8, p. 123-141.

TUCHMAN Gaye, 1973, « Making news by doing work : routinizing the unexpected », American Journal of Sociology, vol. 79, $\mathrm{n}^{\circ}$ 1, p. 110-131.

VAN DE VELDE Danièle, 2000, « Existe-t-il des noms propres de temps ? », Lexique, nº 15, p. 35-45. 


\section{NOTES}

1. Et non, par exemple, comme une incapacité des journalistes, comme dans Fragnon (2007, p. 89) : «[...] l'identification de ces évènements [le 11 septembre 2001 et le 11 septembre 1973 au Chili] par leur seule date renvoie à une dénomination minimale du fait historique et montre par là-même l'incapacité des énonciateurs à trouver une autre dénomination pertinente ».

2. Nous entendons par évènement un ensemble de faits d'intérêt public identifiés comme un tout unique et non répétable. Par ailleurs, contrairement au fait divers, il porte un nom (ou plusieurs, lorsqu'il se décline en un paradigme désignationnel selon le concept de Mortureux, 1993) qui assure sa traçabilité au long du temps.

3. Nous appelons ainsi les mots qui contiennent des sèmes d'évènementialité, et qui s'articulent avec un complément pour désigner un évènement : l'affaire du voile, la guerre en Irak, etc.

4. Nous sommes consciente de la difficulté de nommer cet objet en suivant les règles de composition des mots en -onyme. En effet, comment désigner un nom de temps en fonction évènementielle ? La racine chronos étant réservée (voir infra) à la désignation des périodes, il nous fallait trouver une nouvelle base de dérivation (en l'occurrence, héméra). Cependant, la question de la dimension évènementielle des noms de temps n'est pas résolue pour autant. Comment composer un mot qui superpose la dimension temporelle et la dimension évènementielle ? Étant donné que notre catégorisation n'est pas référentielle (par type d'évènement) mais linguistique (par type de désignant), nous avons choisi une dénomination métalinguistique (nom d'évènement formé à partir d'une date).

5. Cet article a été rédigé avant la publication, dans le numéro 87 de notre revue, d'un dossier consacré à l'étude des chrononymes (NDLR).

6. Parmi les auteurs qui suivent cette démarche : Morin (1972, p. 18), qui distingue évènements " autogénérés ( (développés selon une logique interne) et "hétérogénérés » (qui ont besoin d'une incitation externe), ou encore Molotch et Lester (1996), qui classent l'évènement selon qu'il est un fait de routine, un accident, un scandale ou un heureux hasard (serendipity). Pour sa part, Tuchman (1973) classe l'évènement selon le type de nouvelles (news) qu'il produit : hard news, soft news, spot news, developing news, continuing news.

7. Lorsque nous parlerons du désignant, nous le marquerons par des italiques, tandis que pour parler de l'évènement, nous ne marquerons pas le syntagme.

8. Les façons de désigner un évènement dans la presse ne sont pas infinies, mais bien prédéterminées par le discours journalistique en général et les contraintes techniques en particulier (et surtout spatiales, dans la mesure où le lieu d'irruption de l'évènement dans la presse écrite est le titre). Notre classement des désignants repose sur une vue d'ensemble de cette grille « préétablie ».

9. Nos hypothèses de travail étant liées à la langue de production des désignants évènementiels, la plupart de nos exemples sont tirés de quotidiens et d'hebdomadaires français ou de leurs versions électroniques: Le Monde, Libération, L'Humanité, Courrier international et Le Nouvel Observateur de 1995 à 2008.

10. Les cyclones, par exemple, portent des prénoms de personne depuis le début $\mathrm{du} 20^{\mathrm{e}}$ siècle pour faciliter leur identification. Ces noms font partie d'une liste prédéterminée qui peut revenir régulièrement, à moins qu'il s'agisse d'un phénomène particulièrement meurtrier, auquel cas le nom est retiré de la liste (voir le site du National Hurricane Center : http://www.nhc.noaa.gov).

11. Même si, comme nous l'avons observé ailleurs (Calabrese, à paraitre/a), la motivation du désignant, en tant qu'attribution d'un statut à celui-ci, est toujours un symptôme de notre conception politique ou sociale des évènements.

12. Paradoxalement, c'est l'instabilité du désignant qui lui permet de se figer rigidement à un référent (pour l'instabilité constitutive des désignants évènementiels, voir Calabrese, à paraitre/ b). 
13. Selon le dictionnaire de l'Atilf, le mot (qui signifie « vague d'orage » en japonais) est attesté en français dès 1927.

14. Selon Flaux (2000, p. 1233), « un Npa [nom propre en antonomase] est un Np employé pour désigner un référent autre que son porteur initial, sur la base d'une similitude reconnue entre les propriétés du référent visé et celles du porteur initial. Pour qu'un Np fonctionne comme un Npa il faut, mais il ne suffit pas, que le porteur initial jouisse d'une certaine notoriété, cette condition ne valant pas, bien sûr, pour les antonomases discursives. Les antonomases ne sont pas réservées aux $\mathrm{Np}$ de personnes. Les $\mathrm{Np}$ de lieux et de temps peuvent aussi être employés de manière antonomasique ».

15. Par ailleurs, tous les grands évènements n'ont pas de date commémorative, comme c'est le cas pour Tchernobyl, qui revient plus ou moins souvent grâce aux dossiers qu'y consacrent les journaux. D'autres, plus prégnants par leur profondeur historique et leurs conséquences dans notre présent, comme la Shoah (avec son désignant évènementiel correspondant), ont des dates commémoratives qui ne recoupent pas l'évènement désigné, comme le 27 janvier, date retenue par le Conseil de l'Europe pour la Journée internationale de commémoration en mémoire des victimes de l'holocauste (jour de la libération d'Auschwitz), ou le 4 mai (Yom HaShoah), qui commémore le soulèvement du ghetto de Varsovie.

16. Mais même si, pour des raisons statistiques, les héméronymes entrent rarement en concurrence au niveau local, cela peut toutefois arriver, comme c'est le cas pour le 9 novembre en Allemagne, qui désigne « la proclamation de la république parlementaire [...], la tentative de putsch d'Adolf Hitler à Munich en 1923, les pogromes antisémites de 1938 et la chute du mur de Berlin en $1989 »$ (Schubert).

\section{RÉSUMÉS}

Cet article avance la catégorie d'héméronyme, qui a pour but de décrire les désignants d'évènements formés à partir d'une date (le 11 septembre, le 21 avril) dans le discours médiatique. Nous essayons d'exposer leur comportement spécifique en discours, ainsi que leur fonctionnement par rapport aux autres types de désignants évènementiels (les toponymes, par exemple). L'analyse met en avant le rapport entre la morphologie du désignant (une date) et sa capacité à emmagasiner la mémoire des faits.

This paper puts forward the category of hemeronym, which intends to describe date-based event names $(9-11,21$ st April) in media discourse. We try to show their specific discursive behaviour, and to compare them to other event names (eg toponyms). The analysis points out the connection between the morphology of the name (a date) and its ability to store the historical memory.

Este artículo propone la categoría de hemerónimo, que tiene como objetivo describir los designadores de acontecimiento construidos a partir de una fecha. Intentamos explicar su comportamiento específico en discurso, así como su funcionamiento con respecto a otros tipos de designadores de acontecimiento (los topónimos, por ejemplo). El análisis plantea la relación que existe entre la morfología del designador (una fecha) y su capacidad para almacenar la memoria histórica. 
INDEX

Palabras claves : acontecimiento, designaciones mediáticas, designadores de acontecimiento, hemerónimo vs cronónimo, nombre propio

Mots-clés : désignant évènementiel, désignation médiatique, évènement, héméronyme vs chrononyme, nom propre

Keywords : event, event-names, hemeronym vs chrononym, media designations, proper name

\section{AUTEUR}

\section{LAURA CALABRESE STEIMBERG}

Université libre de Bruxelles / Ladisco

Laura.Marina.Calabrese@ulb.ac.be 